\title{
Neutrino lines from DM decay induced by high-scale seesaw interactions
}

\author{
Rupert Coy and Thomas Hambye \\ Service de Physique Théorique, Université Libre de Bruxelles, \\ Boulevard du Triomphe, CP225, 1050 Brussels, Belgium \\ E-mail: rupert.coy@ulb.ac.be, thambye@ulb.ac.be
}

ABSTRACT: If the stability of the dark matter (DM) particle is due to an accidental symmetry, nothing prevents UV physics from destabilising it by inducing DM decays suppressed by powers of the UV scale. The seesaw physics, presumably at the origin of neutrino mass, could induce such a decay. We show that if the seesaw scale lies around the usual Weinberg operator scale, the induced DM decay could generically lead to neutrino lines whose intensity is of the order of the present sensitivity of neutrino telescopes. We illustrate this possibility with models in which the DM is made of the gauge boson(s) of an abelian or non-abelian gauge symmetry.

KeYwords: Beyond Standard Model, Cosmology of Theories beyond the SM, Gauge Symmetry, Neutrino Physics

ArXiv EPrint: 2012.05276 


\section{Contents}

1 Introduction 1

2 A simple setup 3

$\begin{array}{llr}3 & \text { Results } & 8\end{array}$

4 Comparison with other seesaw induced DM decay setups 10

5 Non-abelian case $\quad 12$

6 Summary 12

\section{Introduction}

The four stable particles that exist in the Standard Model (SM) are all stable for a fundamental reason, related to Lorentz invariance, the gauge symmetries of the SM and the quantum numbers of the SM particles under these gauge symmetries. The evidence for a fifth (or more) stable particle, the dark matter (DM) particle(s), raises the question of whether its stability hides a new fundamental symmetry/principle, for instance a new gauge symmetry, rather than just an (often assumed) ad hoc discrete symmetry (see e.g. [1]). Among various stabilisation mechanisms, the possibility that the DM particle(s) would be stable due to an accidental symmetry is rather intriguing. Various frameworks of this type can be considered. One option is simply to assume that DM belongs to a large enough weak multiplet that no renormalisable interactions that could destabilise it can be written down [2]. Another option consists of assuming a new gauge symmetry whose breaking leaves an accidental symmetry which is not a subgroup of the gauge symmetry. This can be done on the basis of an abelian [3, 4] or non-abelian gauge symmetry [3, 5-10]. Other possibilities of course do exist.

If DM is accidentally stable, nothing forbids some UV physics, lying at the scale $\Lambda_{\mathrm{UV}}$, from destabilising it. This is similar to what is expected for the proton, for instance, in GUT theories. The DM lifetime must obviously be longer than the age of the universe, $\sim 10^{18}$ sec, or in fact much longer, $\tau_{\mathrm{DM}} \gtrsim 10^{22-29} \mathrm{sec}$, in order not to produce fluxes of cosmic rays larger than those observed (assuming decays into SM particles, depending on the decay channel and DM mass, and considering here $\left.m_{\mathrm{DM}} \gtrsim 1 \mathrm{GeV}\right)$. If the UV physics induces a decay amplitude which is suppressed by only one power of the UV scale, the DM decay width induced is typically proportional to $(1 / 8 \pi) \cdot m_{\mathrm{DM}}^{3} / \Lambda^{2}$, and it is known that this is many orders of magnitude too fast to fulfil these constraints (for $\Lambda$ no larger than the Planck scale). Tiny couplings are necessary in this case, so that $\Lambda$ is an effective scale 
quite different from the much lower fundamental scale $\Lambda_{\mathrm{UV}}$, i.e. $\Lambda=\Lambda_{\mathrm{UV}} / g$, where $g \ll 1$ is some combination of couplings. Instead, a decay amplitude suppressed by two powers of the UV scale, $\Lambda=\Lambda_{\mathrm{UV}}$, gives a decay width proportional to $(1 / 8 \pi) \cdot m_{\mathrm{DM}}^{5} / \Lambda_{\mathrm{UV}}^{4}$. For DM mass of order the electroweak scale and $\Lambda_{\mathrm{UV}}$ of order the GUT scale, this nicely leads to lifetimes of order the lower bound from cosmic rays. ${ }^{1}$ In this case, there is the possibility of a direct connection between the fundamental UV scale and the DM lifetime. Besides neutrino mass and proton decay probes, this provides another nice avenue to study very high scale physics, which we investigate in this letter.

The existence of non-vanishing neutrino masses is the most established (in particular from laboratory experiments) evidence for beyond the standard model physics, and the seesaw mechanism constitutes their most attractive explanation. Thus, invoking seesaw physics to destabilise the DM particle is a well-motivated possibility. Although the seesaw states, for instance right-handed neutrinos $N_{i}$ in the type-I seesaw model, could lie at a low scale, clearly the smallness of the neutrino masses fits very well with these seesaw states being at a much higher scale than the electroweak scale, not far from the GUT scale. For seesaw Yukawa couplings of order unity, the seesaw scale is the scale of the $L L H H / \Lambda_{W}$ Weinberg operator, $\Lambda_{W} \sim 10^{15} \mathrm{GeV}$. The seesaw interactions are not necessarily expected to cause relevant DM decays. For instance, adding right-handed neutrinos to the minimal DM quintuplet $\psi_{\mathrm{DM}}^{(5)}$ setup [2] doesn't easily induce a decay of this quintuplet. ${ }^{2}$ However, there are other models where the seesaw is expected to cause such a decay, see [11-21] and below. In particular, if the DM, and more generally its associated sector, is comprised of SM singlet particles, the right-handed neutrinos can easily couple to this sector, also being SM singlets. This allows the decay DM $\rightarrow N^{*}+X$, with a further conversion of the virtual right-handed neutrino, $N^{*}$, into a SM neutrino through seesaw mixing, thereby allowing DM to decay into neutrinos. The seesaw interactions therefore not only offer the possibility of inducing a slow DM decay, but also a way of easily producing SM neutrino(s) in the final state, in particular a neutrino line if the decay is to a two-body final state. As is well known, monochromatic $\gamma$ [22-28] or neutrino [18, 29-32] signals are "DM smoking guns" because there is basically no astrophysical background for such a signal.

From the discussion above, it is clear that if, through the exchange of a heavy seesaw state, an operator (that is, a decay amplitude) suppressed by only one power of the seesaw scale is generated, the decay will naturally be far too fast, unless the DM mass scale is quite low (well below the GeV scale) and/or this seesaw exchange diagram involves small couplings or extra tiny mass ratios. In all these ways out, the direct connection between the Weinberg operator scale and the DM lifetime is lost. This situation occurs for instance in Majoron DM models [11-17, 19, 20, 33, 34], in which the decay amplitude into a pair of charged leptons, suppressed by only one power of the seesaw scale, is induced at the one-loop level. Another example of this situation was recently considered in [21]. However,

\footnotetext{
${ }^{1}$ For instance, for $m_{\mathrm{DM}}=100 \mathrm{GeV}$ and $\Lambda=10^{15} \mathrm{GeV}$, one gets $\tau_{\mathrm{DM}} \sim 10^{27}$ sec. More generally, this holds replacing $m_{\mathrm{DM}}^{5}$ in the decay width with any dimension- 5 combination of masses around the electroweak scale.

${ }^{2}$ The lowest-dimensional operator involving both fermions is $\bar{N} H^{4} \psi_{\mathrm{DM}}^{(5)} / \Lambda^{3}$, which from the exchange of a right-handed neutrino leads to the dimension- 8 operator, $\bar{L} H^{5} \psi_{\mathrm{DM}}^{(5)} /\left(\Lambda^{3} m_{N}\right)$.
} 
if a model manages to not induce any decay amplitude suppressed by one power of the seesaw scale, but does induce an amplitude suppressed by two powers of this scale, the direct connection between neutrino mass and the DM lifetime can hold. This moreover leads to a neutrino line with intensity of the order of the sensitivity of present indirect detection experiments. This is the possibility we consider in this work.

\section{A simple setup}

The example model we will consider in detail assumes an extra $\mathrm{U}(1)_{X}$ gauge symmetry spontaneously broken by the vacuum expectation value of a scalar boson, $\phi$, with the addition of a vector-like fermion charged under it, $\chi \cdot^{3}$ The associated Lagrangian is

$$
\mathcal{L}=\mathcal{L}_{\mathrm{SM}}-\frac{1}{4} F_{\mu \nu}^{X} F^{X \mu \nu}+\bar{\chi}\left(i \not D-m_{\chi}\right) \chi+D_{\mu} \phi^{\dagger} D^{\mu} \phi-\lambda_{m} \phi^{\dagger} \phi H^{\dagger} H-V(\phi),
$$

where $D_{\mu}=\partial_{\mu}-i g_{X} Q_{X} A_{\mu}^{\prime}, V(\phi)=\mu^{2} \phi^{\dagger} \phi+\lambda_{\phi}\left(\phi^{\dagger} \phi\right)^{2}$, and $F_{\mu \nu}^{X}$ is the $\mathrm{U}(1)_{X}$ field strength tensor. Here we assume that there is no kinetic mixing interaction between the $\mathrm{U}(1)_{X}$ and hypercharge gauge bosons. We parameterise the scalar by $\phi=\left(\eta^{\prime}+v_{\phi}\right) / \sqrt{2}$, with $v_{\phi}=\sqrt{-2 \mu^{2} / \lambda_{\phi}}$, the NGB from the spontaneously broken $\mathrm{U}(1)_{X}$ being eaten by the $A^{\prime}$. Without the fermion $\chi$, this is the DM model of refs. $[3,4]$, where the $\mathrm{U}(1)_{X}$ gauge boson, $A^{\prime}$, is the DM candidate. It is stable because after spontaneous breaking, the model displays an accidental $\mathbb{Z}_{2}$ symmetry under which the gauge boson is odd. ${ }^{4}$ Adding the extra fermion, $\chi$, leads to two possible DM patterns. If $m_{A^{\prime}}=g_{X} v_{\phi}>2 m_{\chi}$, the vector boson decays into a pair of fermions and is not stable anymore, thanks to the fact that the fermion-gauge boson interaction breaks the $\mathbb{Z}_{2}$ symmetry. But the $\chi$ is stable because a $\mathbb{Z}_{2}$ symmetry under which $\chi$ is odd remains, due to Lorentz invariance and the fact that it is charged under $\mathrm{U}(1)_{X}$. If instead $m_{A^{\prime}}<2 m_{\chi}$, a multi-component DM setup arises wherein both the $A^{\prime}$ and $\chi$ are stable, even though the remnant $\mathbb{Z}_{2}$, under which $A^{\prime}$ is odd, is broken. Here we focus on how DM can be destabilised in the latter framework by extra right-handed neutrinos. ${ }^{5}$ Adding these seesaw states opens up the possibility of neutrino portal interactions,

$$
\delta \mathcal{L}=-\left(Y_{L} \overline{N_{R}} \phi \chi_{L}+Y_{R} \overline{N_{R}^{c}} \phi \chi_{R}+\text { h.c. }\right)
$$

on top of the usual seesaw interactions,

$$
\mathcal{L}_{\text {seesaw }}=i \overline{N_{R}} \not \partial N_{R}-\frac{1}{2} m_{N}\left(\overline{N_{R}} N_{R}^{c}+\overline{N_{R}^{c}} N_{R}\right)-\left(Y_{\nu} \overline{N_{R}} \tilde{H}^{\dagger} L+\text { h.c. }\right) .
$$

\footnotetext{
${ }^{3} \mathrm{~A}$ more involved chiral fermion structure in which the fermions acquire their mass from the spontaneous breaking of a gauge symmetry could also be considered.

${ }^{4}$ Actually, this charge conjugation symmetry of the abelian case is not fully accidental here since it holds only if one assumes no kinetic mixing, which somewhat goes against the general spirit of our introduction above. To avoid that, one can go to the non-abelian case, which has very similar phenomenology to what we are interested in for this work. For simplicity, we will first stick to the abelian case and will comment on the non-abelian one later.

${ }^{5}$ The vector DM relic density production can proceed in a thermal or non-thermal way, in particular through the Higgs portal and $\mathrm{U}(1)_{X}$ interactions, see $[3-6,35]$. As for the $\chi$ particles, they are produced by $\mathrm{U}(1)_{X}$ interactions, but at the end of the day this will not turn out to be important since these particles are destabilised by seesaw interactions and are not part of DM today, see below.
} 
Here we consider only one right-handed neutrino and one SM lepton doublet, $L$. The generalisation to several flavours is straightforward. In eq. (2.2), the $Y_{L, R}$ neutrino portal interactions are allowed if the $\phi \chi$ field combination is neutral under $\mathrm{U}(1)_{X}$, so in the following we will assume $Q_{\chi}=-Q_{\phi}=1 .{ }^{6}$ Note that $Y_{L}, Y_{R}$, and $Y_{\nu}$ can all be made real and positive by rephasing appropriately the $\chi_{L}, \chi_{R}$, and $L$ fields. Note also that the vectorlike character of the new $\chi_{L, R}$ fermions ensures that the model is free of SM and $\mathrm{U}(1)_{X}$ gauge anomalies.

For a heavy right-handed neutrino, where $m_{N} \gg m_{A^{\prime}}, m_{\chi}$, the $\chi \rightarrow N \phi$ decays are kinematically forbidden, but $Y_{L, R}$ induces $\chi \rightarrow \nu_{L} \phi$ decays through seesaw mixing. Similarly, the $Y_{L, R}$ interactions and seesaw mixing induce $A^{\prime} \rightarrow \nu_{L} \bar{\nu}_{L}$ decays, see figure 1 . The amplitude of the first process is suppressed by one power of $m_{N}$ because it involves one seesaw mixing. The second process instead involves two seesaw mixings and hence is suppressed by two powers of $m_{N}$. Thus, the second process can generically lead to neutrino lines with an intensity of order the present experimental sensitivity, whereas the first one gives a lifetime much smaller than the age of the Universe unless $Y_{L . R}$ are tiny.

To compute the decay amplitudes of both processes it is necessary to go to the mass eigenstate basis for the four neutral leptons, $\nu_{L}, N_{R}, \chi_{L}, \chi_{R}$, and the scalar bosons. The neutral lepton mass Lagrangian is

$$
\mathcal{L}_{\text {mass }}=-\frac{1}{2}\left(\overline{\nu_{L}^{c}} \overline{\chi_{L}^{c}} \overline{\chi_{R}} \overline{N_{R}}\right)\left(\begin{array}{cccc}
0 & 0 & 0 & m \\
0 & 0 & m_{\chi} & m_{L} \\
0 & m_{\chi} & 0 & m_{R} \\
m & m_{L} & m_{R} & m_{N}
\end{array}\right)\left(\begin{array}{c}
\nu_{L} \\
\chi_{L} \\
\chi_{R}^{c} \\
N_{R}^{c}
\end{array}\right)+h . c .,
$$

where $m=v Y_{\nu} / \sqrt{2}$ and $m_{L, R}=v_{\phi} Y_{L, R} / \sqrt{2}$. The mass eigenstates, $n_{i}=\left(\begin{array}{lll}\nu & \chi_{1} & \chi_{2}\end{array}\right)^{T}$, are related to the gauge eigenstates by

$$
\left(\begin{array}{c}
\nu_{L}+\nu_{L}^{c} \\
\chi_{L}+\chi_{L}^{c} \\
\chi_{R}+\chi_{R}^{c} \\
N_{R}+N_{R}^{c}
\end{array}\right) \simeq O\left(\begin{array}{c}
\nu \\
\chi_{1} \\
\chi_{2} \\
N
\end{array}\right)
$$

with $O$ given by

$$
\left(\begin{array}{cccc}
i & -\frac{m\left(m_{L}+m_{R}\right)}{\sqrt{2} m_{\chi} m_{N}} & \frac{i m\left(m_{R}-m_{L}\right)}{\sqrt{2} m_{\chi} m_{N}} & \frac{m}{m_{N}} \\
\frac{i m m_{R}}{m_{\chi} m_{N}} & \frac{1}{\sqrt{2}}+\frac{m_{R}^{2}-m_{L}^{2}}{4 \sqrt{2} m_{\chi} m_{N}} & -\frac{i}{\sqrt{2}}+\frac{i\left(m_{R}^{2}-m_{L}^{2}\right)}{4 \sqrt{2} m_{\chi} m_{N}} & \frac{m_{L}}{m_{N}} \\
\frac{i m_{L} m_{L}}{m_{\chi} m_{N}} & \frac{1}{\sqrt{2}}-\frac{m_{R}^{2}-m_{L}^{2}}{4 \sqrt{2} m_{\chi} m_{N}} & \frac{i}{\sqrt{2}}+\frac{i\left(m_{R}^{2}-m_{L}^{2}\right)}{4 \sqrt{2} m_{\chi} m_{N}} & \frac{m_{R}}{m_{N}} \\
\frac{-i m}{m_{N}} & -\frac{m_{L}+m_{R}}{\sqrt{2} m_{N}} & -\frac{i\left(m_{R}-m_{L}\right)}{\sqrt{2} m_{N}} & 1
\end{array}\right),
$$

at $\mathcal{O}\left(1 / m_{N}\right)$. The mass eigenvalues are $\simeq m^{2} / m_{N}, m_{\chi} \mp\left(m_{L} \pm m_{R}\right)^{2} /\left(2 m_{N}\right)$, and $m_{N}$, respectively.

\footnotetext{
${ }^{6}$ Any Yukawa interaction, including the SM ones, always requires that the U(1) charges of the particles involved "miraculously" sum up to 0.
} 


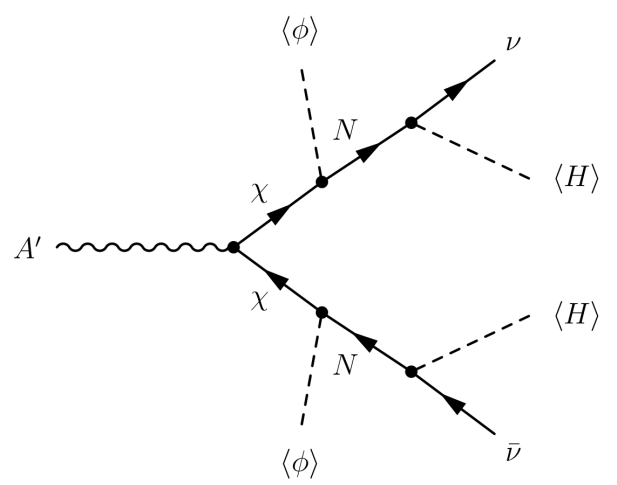

Figure 1. The $A^{\prime} \rightarrow \nu \bar{\nu}$ decay at tree-level.

For the scalar bosons, after SSB the real scalar of the SM Higgs doublet, $h^{\prime}$, and hidden sector scalar boson, $\eta^{\prime}$, mix through the Higgs portal interaction, leading to mass eigenstates,

$$
\left(\begin{array}{l}
h \\
\eta
\end{array}\right)=\left(\begin{array}{cc}
\cos \varphi & -\sin \varphi \\
\sin \varphi & \cos \varphi
\end{array}\right)\left(\begin{array}{l}
h^{\prime} \\
\eta^{\prime}
\end{array}\right)
$$

where the mixing angle is

$$
\tan 2 \varphi=\frac{\lambda_{m} v v_{\phi}}{\lambda_{\phi} v_{\phi}^{2}-\lambda v^{2}}
$$

The mass eigenvalues are

$$
m_{h, \eta}^{2}=\lambda v^{2}+\lambda_{\phi} v_{\phi}^{2} \pm \sqrt{\left(\lambda v^{2}-\lambda_{\phi} v_{\phi}^{2}\right)^{2}+\lambda_{m}^{2} v^{2} v_{\phi}^{2}},
$$

which in the limit of $\lambda_{m} \ll 1$ reduce to $m_{h}^{2} \simeq 2 \lambda v^{2}$ and $m_{\eta}^{2} \simeq 2 \lambda_{\phi} v_{\phi}^{2}$.

The only two-body final state into which the hidden vector DM particle can decay at tree-level is a pair of neutrinos. We find

$$
\Gamma\left(A^{\prime} \rightarrow \nu \bar{\nu}\right)_{\text {tree }} \simeq \frac{g_{X}^{2} Y_{\nu}^{4}\left(Y_{L}^{2}-Y_{R}^{2}\right)^{2} v^{4} v_{\phi}^{4} m_{A^{\prime}}}{96 \pi m_{\chi}^{4} m_{N}^{4}} .
$$

This process is, as we anticipated, suppressed by four powers of the seesaw scale, more precisely by four powers of $\left(Y_{L, R} v_{\phi} / m_{\chi}\right)\left(Y_{\nu} v / m_{N}\right)$, as it requires two $\chi \rightarrow N_{R} \rightarrow \nu_{L}$ transitions, see figure 1. Note that when $Y_{L}=Y_{R}$, the decay width of eq. (2.10) vanishes at $\mathcal{O}\left(1 / m_{N}^{4}\right)$ as the diagrams with intermediate $\chi_{L}$ and $\chi_{R}$ involve a relative negative sign.

When $m_{A^{\prime}}$ is above the EW scale, many three-body and four-body decays open up by replacing Higgs vev insertions with physical particles in the final state. The possible three-body decays are $A^{\prime} \rightarrow \nu \bar{\nu} h, A^{\prime} \rightarrow \nu \bar{\nu} Z$, and $A^{\prime} \rightarrow \nu \ell^{ \pm} W^{\mp}$. The allowed four-body decays can easily be deduced. Neglecting the final state masses, the rates are

$$
\begin{aligned}
\Gamma_{A^{\prime}, \text { three-body }} & \simeq \frac{3 g_{X}^{2} Y_{\nu}^{4}\left(Y_{L}^{2}-Y_{R}^{2}\right)^{2} v^{2} v_{\phi}^{4} m_{A^{\prime}}^{3}}{64(4 \pi)^{3} m_{\chi}^{4} m_{N}^{4}} \\
\Gamma_{A^{\prime}, \text { four-body }} & \simeq \frac{g_{X}^{2} Y_{\nu}^{4}\left(Y_{L}^{2}-Y_{R}^{2}\right)^{2} v_{\phi}^{4} m_{A^{\prime}}^{5}}{320(4 \pi)^{5} m_{\chi}^{4} m_{N}^{4}} .
\end{aligned}
$$



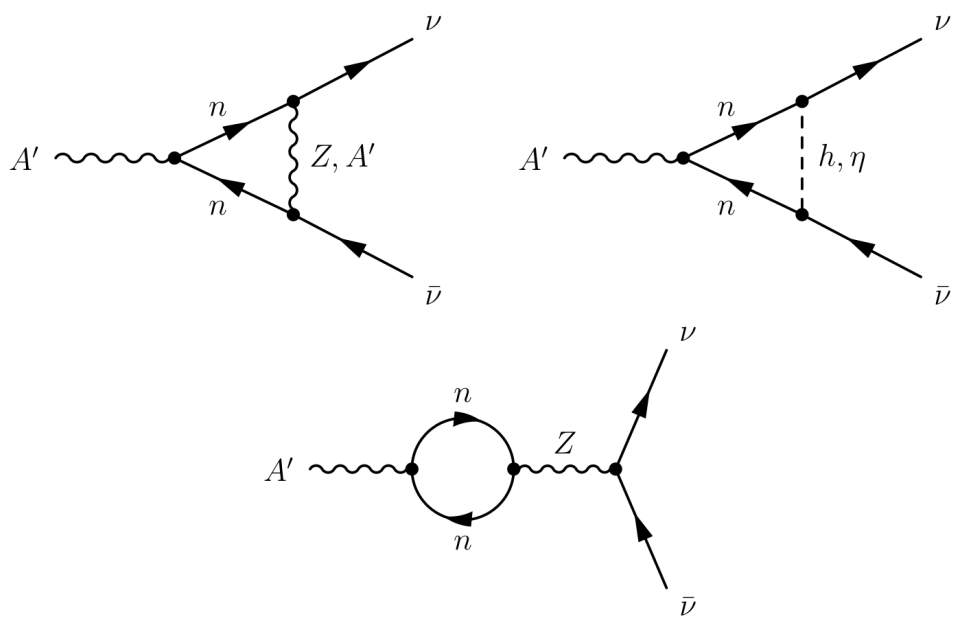

Figure 2. The one-loop diagrams giving $A^{\prime} \rightarrow \nu \bar{\nu}$ decay. Here $n=\nu, \chi_{1,2}, N$ are mass eigenstates.

We see that the phase space suppression compared to the two-body decay is compensated by additional powers of $m_{A^{\prime}} / v$, so that the three-body rate is larger than the two-body rate for $m_{A^{\prime}} \gtrsim 2.9 \mathrm{TeV}$ and the four-body rate becomes dominant for $m_{A^{\prime}} \gtrsim 12 \mathrm{TeV}$. On the other hand, replacing $\phi$ vev insertions gives factors of $m_{A^{\prime}} / v_{\phi} \lesssim 1$ while paying the price of the phase space suppression, so these decays are subdominant and can be neglected.

One-loop decay processes also have to be considered. The decay to neutrinos, figure 2, proceeds through the exchange of a scalar or vector boson in the $t$-channel or through oneloop $A^{\prime}-Z$ mixing. The $Z$ exchange diagram dominates, and for $m_{A^{\prime}, \chi} \gg m_{h}$, we have

$$
\mathcal{M} \simeq \frac{g_{X} Y_{\nu}^{2}\left(Y_{L}^{2}-Y_{R}^{2}\right) v_{\phi}^{2}}{128 \pi^{2} m_{N}^{2}} \log \frac{m_{N}^{2}}{m_{\chi}^{2}} \bar{u}\left(p_{\nu}\right) \gamma_{\mu} \gamma_{5} v\left(p_{\bar{\nu}}\right) \epsilon^{\mu}\left(p_{A^{\prime}}\right),
$$

plus terms not enhanced by the large log. This leads to

$$
\Gamma\left(A^{\prime} \rightarrow \nu \bar{\nu}\right)_{\text {loop }} \simeq \frac{g_{X}^{2} Y_{\nu}^{4}\left(Y_{L}^{2}-Y_{R}^{2}\right)^{2} v_{\phi}^{4} m_{A^{\prime}}}{96(4 \pi)^{5} m_{N}^{4}} \log ^{2} \frac{m_{N}^{2}}{m_{\chi}^{2}},
$$

when the tree-level contribution can be neglected. ${ }^{7}$ This rate is suppressed by four powers of the $\chi-\nu_{L}$ mixing and there are no extra powers of $m_{N}$ in the numerator coming from the fermionic trace or loop integral. Thus, it is of the same order in $1 / m_{N}$ as the contributions of eqs. (2.10)-(2.12). Similarly to the point emphasised in [21], since the two-body decay is proportional to powers of vacuum expectations values, then for DM masses well beyond the values of these vevs, the one-loop contribution can be greater than the tree-level one. This stems from the fact that the loop contribution involves the propagators of the scalar fields, rather than their vevs. As a result, with respect to the tree-level contributions of eq. (2.10), the loop factor is compensated by a factor of $m_{\chi}^{4} / v^{4}$. For instance, for $m_{A^{\prime}}=m_{\chi}$, the rate in eq. (2.14) is larger than the tree-level width given in eq. (2.10) for $m_{A^{\prime}} \gtrsim$

\footnotetext{
${ }^{7}$ We will not give here the explicit form of the (constructive) tree-level and one-loop interference term but take it into account in our results below.
} 

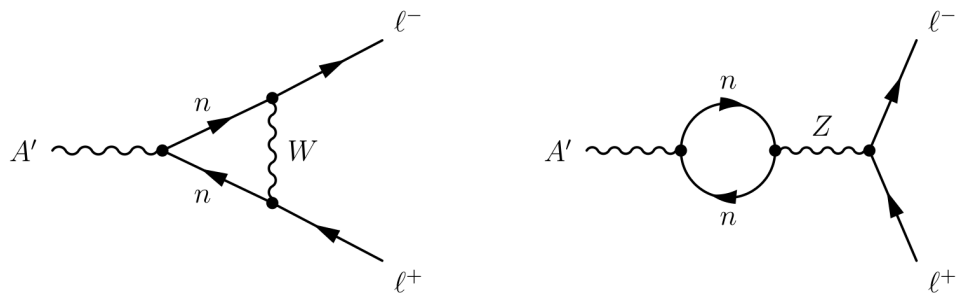

Figure 3. The one-loop diagrams giving $A^{\prime} \rightarrow \ell^{-} \ell^{+}$decay. Here $n=\nu, \chi_{1,2}, N$ are mass eigenstates.

1.6 TeV. Comparing with the four-body decays, we have $\Gamma\left(A^{\prime} \rightarrow \nu \bar{\nu}\right)_{\text {loop }} / \Gamma_{A^{\prime} \text {,four-body }} \simeq$ $(10 / 3)\left(m_{\chi} / m_{A^{\prime}}\right)^{4} \log ^{2}\left(m_{N}^{2} / m_{A^{\prime}}^{2}\right)$, hence the two-body decay dominates. Thus the fourbody contribution can always be neglected, as can the three-body one.

The decay to charged leptons, shown in figure 3, proceeds either from the exchange of a $W$ in the $t$-channel or through $A^{\prime}-Z$ one-loop mixing. Due to $\mathrm{SU}(2)_{L}$ symmetry, the leading order amplitude for this process is the same as the amplitude for the loop-level decay to neutrinos, (neglecting the final state lepton masses), and hence the partial width $\Gamma\left(A^{\prime} \rightarrow \ell^{+} \ell^{-}\right)$is the same as the width in eq. (2.14).

Finally, we note that one-loop decays to bosonic final states, such as $A^{\prime} \rightarrow Z h$ and $A^{\prime} \rightarrow W^{+} W^{-}$, also exist for sufficiently heavy $A^{\prime}$, with comparable rates to $A^{\prime} \rightarrow \ell^{+} \ell^{-}$. We will not consider their contributions as they do not bring any spectral features and do not change by much the constraints one can obtain from diffuse fluxes of cosmic rays.

Unlike for the hidden vector decay, the decay of the fermion $\chi$ is suppressed by only two powers of $m_{N}$ since it involves only one $\chi \rightarrow N \rightarrow \nu$ transition. There are many possible decay channels. In the limit of $\varphi \simeq 0$, the decay widths to $\eta \nu$ and $h \nu$ are

$$
\Gamma\left(\chi_{1,2} \rightarrow \eta \nu\right) \simeq \frac{Y_{\nu}^{2}\left(Y_{L} \mp Y_{R}\right)^{2} v^{2} m_{\chi}}{64 \pi m_{N}^{2}}\left(1-\frac{m_{\eta}^{2}}{m_{\chi}^{2}}\right)^{2}
$$

and

$$
\Gamma\left(\chi_{1,2} \rightarrow h \nu\right) \simeq \frac{Y_{\nu}^{2}\left(Y_{L} \pm Y_{R}\right)^{2} v_{\phi}^{2} m_{\chi}}{64 \pi m_{N}^{2}}\left(1-\frac{m_{h}^{2}}{m_{\chi}^{2}}\right)^{2} .
$$

There are also decays to SM gauge bosons, when kinematically allowed, with partial widths

$$
\begin{aligned}
\Gamma\left(\chi_{1,2} \rightarrow W^{ \pm} \ell^{\mp}\right) & \simeq \frac{Y_{\nu}^{2}\left(Y_{L} \pm Y_{R}\right)^{2} v_{\phi}^{2} m_{\chi}}{64 \pi m_{N}^{2}} f\left(m_{W}^{2} / m_{\chi}^{2}\right), \\
\Gamma\left(\chi_{1,2} \rightarrow Z \nu\right) & \simeq \frac{Y_{\nu}^{2}\left(Y_{L} \pm Y_{R}\right)^{2} v_{\phi}^{2} m_{\chi}}{64 \pi m_{N}^{2}} f\left(m_{Z}^{2} / m_{\chi}^{2}\right),
\end{aligned}
$$

where $f(x)=(1-x)^{2}(1+2 x)$. Finally, the $\chi$ also decays to $A^{\prime} \nu$, with partial width

$$
\Gamma\left(\chi_{1,2} \rightarrow A^{\prime} \nu\right) \simeq \frac{Y_{\nu}^{2}\left(Y_{L} \mp Y_{R}\right)^{2} v^{2} m_{\chi}}{64 \pi m_{N}^{2}} f\left(m_{A^{\prime}}^{2} / m_{\chi}^{2}\right) .
$$

If $m_{\chi}<m_{\eta}, m_{A^{\prime}}, m_{W}$ the leading decays are to three SM fermions, mediated by the $W$ or $Z$ boson, which are also suppressed by two powers of $m_{N}$. 


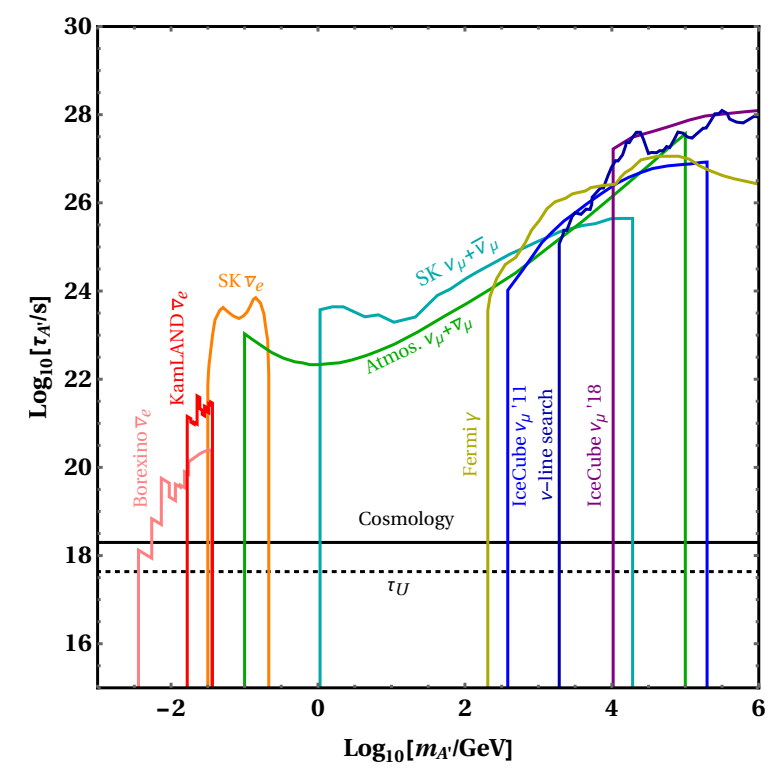

Figure 4. Bounds on the lifetime of dark matter assuming it decays only into $\nu \bar{\nu}$. Here we assume that the DM couples universally to the three neutrinos flavours.

\section{Results}

Experimental constraints on the lifetime of DM decaying into a pair of neutrinos can be found in refs. [20,30,36-40]. The best bounds from DM indirect detection observations are from direct searches of a flux of neutrinos, including those by Borexino [41], KamLAND [42], IceCube [30, 39, 40] and Super-Kamiokande [36, 38, 43, 44], or from the search of a diffuse flux of cosmic rays emitted by the neutrinos [45]. Cosmological constraints also exist. Besides the condition that $\tau_{\mathrm{DM}}>\tau_{U}$, CMB data gives $\tau_{\mathrm{DM}}>4.6 \tau_{U}$ [46]. In ref. [20], many of these constraints were compiled and translated into an upper bound on the $\mathrm{U}(1)_{B-L}$ breaking scale as a function of the Majoron mass. Translating them back into constraints on the DM lifetime, and adding the dedicated search for neutrino lines from IceCube data [30], recent IceCube collaboration limits [39, 40] and an analysis of Fermi-LAT gammaray data [45], figure 4 shows the various constraints on the DM lifetime. The result shown assumes flavour universality, i.e. $\Gamma\left(A^{\prime} \rightarrow \nu_{\alpha} \bar{\nu}_{\alpha}\right)$ is the same for $\alpha=e, \mu, \tau$ (in this case the neutrino mass hierarchy plays no role). Modifying the branching ratios to each flavour or the neutrino mass hierarchy only mildly affects the results.

In the following, to present the results, we will take a simple benchmark case where the couplings are equal to unity, $g_{X}=Y_{\nu}=\left|Y_{L}^{2}-Y_{R}^{2}\right|=1$, and $m_{\chi}=m_{A^{\prime}}$. This implies $m_{\mathrm{DM}}=v_{\phi}$ and gives $\Gamma\left(A^{\prime} \rightarrow \nu \bar{\nu}\right)=(1 / 96 \pi) \cdot v^{4} m_{A^{\prime}} / m_{N}^{4}$ at tree-level. Figure 5 gives, for this straightforward case, the lower bound on $m_{N}$ we get from the various constraints on the lifetime in figure 4. Again, it is assumed that the DM decays in a flavour-universal way. As expected, the values are typically of order the Weinberg operator scale when $m_{\mathrm{DM}}$ is of order the electroweak scale. Of course, nothing guarentees that $m_{\chi}$ must necessarily be of order $v_{\phi}$, since these two scales are independent in the setup we consider. For $v_{\phi} \neq m_{\chi}$ 


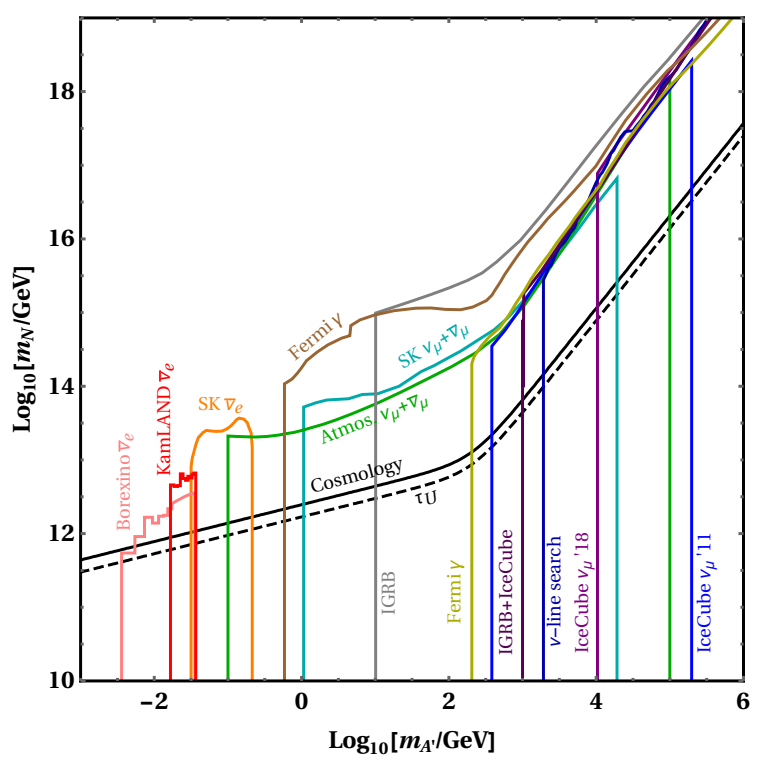

Figure 5. Lower bounds on the heavy sterile neutrino mass, $m_{N}$, from constraints on the lifetime of DM which decays as $A^{\prime} \rightarrow \nu \bar{\nu}$ and $A^{\prime} \rightarrow \ell^{+} \ell^{-}$. Limits on the former are shown in figure 4, while the brown, mustard and grey lines correspond to constraints on the latter from refs. [45, 47, 48], respectively. Here we assume that the couplings are of order unity and $m_{\chi}=m_{A^{\prime}}$, and that the DM couples universally to the three flavours.

and small $m_{A^{\prime}}$, such that the tree-level part of the $A^{\prime} \rightarrow \nu \bar{\nu}$ amplitude dominates, the bounds on $m_{N}$ have to be simply rescaled by one power of the $v_{\phi} / m_{\chi}$ ratio (assuming still $\left.m_{A^{\prime}}<2 m_{\chi}\right)$. For larger $m_{A^{\prime}}$, when the one-loop contribution dominates, the bound on $m_{N}$ depends only logarithmically on $v_{\phi} / m_{\chi}$. Also, considering couplings smaller than unity clearly leads to a less stringent lower bound on $m_{N}$ than in figure 5 .

Figure 5 also shows the lower bound we get on $m_{N}$ from $A^{\prime} \rightarrow \ell^{+} \ell^{-}$(with $l=e, \mu, \tau$ ), using the results of $[45,47,48]$ obtained from IceCube data [49] and Fermi-LAT data [5052] of the inner-Galaxy gamma-ray spectrum and the isotropic gamma-ray background (IGRB). Comparing this bound with the ones from the neutrino channel, one observes that at the moment charged lepton limits are more stringent for $m_{\mathrm{DM}} \gtrsim 10 \mathrm{GeV}$ by a factor of a few (although this relative factor depends somewhat on the flavour composition of the DM decays and the neutrino mass hierarchy). This is interesting because it means that improving the limits for the neutrino channel by a factor of a few would open the possibility of seeing both an associated flux of neutrinos and charged leptons. As mentioned above, for $m_{\mathrm{DM}} \gtrsim 1.6 \mathrm{TeV}$ the loop contribution dominates and predicts an equal decay width for both channels (similarly to the setup of [21]).

If the doubly seesaw-suppressed decay width of the $A^{\prime}$ is of order the experimental sensitivity, the $\chi$ lifetime is expected to be much smaller than the age of the Universe, since the corresponding decay width of $\chi$ is only singly suppressed by the seesaw scale. In figure 6 the dark blue line gives the lifetime of $\chi_{1,2}$ we get assuming the same benchmark set of parameters as for figure 5 . We restrict ourselves to $m_{\chi}>100 \mathrm{GeV}$, so that the $\chi$ has kinematically allowed two-body decays. As the figure shows, its lifetime is around the age 
of the Universe at the beginning of the BBN epoch, $\tau_{\chi} \sim 1$ sec. Since the $\chi$ decay produces electromagnetically coupled SM particles, BBN typically requires that the lifetime must be smaller than 1 sec.

It is interesting to note that the ratio of the lifetime of DM allowed by indirect detection, $\tau_{\mathrm{DM}} \gtrsim 10^{26-29} \mathrm{sec}$, and the age of the Universe at BBN time, $t_{\mathrm{BBN}} \sim 1 \mathrm{sec}$, is rather similar to the ratio of the neutrino mass scale, $m_{\nu} \sim 0.1 \mathrm{eV}$, and the seesaw scale, $\Lambda \sim 10^{15} \mathrm{GeV}$, which is $m_{\nu} / \Lambda \sim 10^{-25}$. This means that if the decay width of the hidden vector is of order the experimental sensitivity for neutrino lines, being suppressed by four powers of the seesaw scale, particles whose decay is suppressed by two powers of the seesaw scale can have already disappeared by the time of BBN. The ratio of the lifetimes (when the tree-level $A^{\prime}$ decay dominates) scales as $\tau_{\chi} / \tau_{A^{\prime}} \sim\left(Y_{\nu} v / m_{N}\right)^{2}\left(g_{X} Y_{L, R} v v_{\phi} / m_{\chi}^{2}\right)^{2}\left(m_{A^{\prime}} / m_{\chi}\right) \sim$ $\left(m_{\nu} / m_{N}\right) \cdot C$ with $C=\left(g_{X} Y_{L, R} v v_{\phi} / m_{\chi}^{2}\right)^{2}\left(m_{A^{\prime}} / m_{\chi}\right)$. To illustrate the above, one can also write down the lifetime of the $\chi$ from $\chi_{1,2} \rightarrow$ SM channels, in the $m_{\chi} \gg v$ limit, as ${ }^{8}$

$$
\tau_{\chi_{1,2}} \simeq \frac{1 \mathrm{sec}}{Y_{\nu}^{2}\left(Y_{L} \pm Y_{R}\right)^{2}}\left(\frac{1.5 \mathrm{TeV}}{m_{\chi}^{1 / 3} v_{\phi}^{2 / 3}}\right)^{3}\left(\frac{m_{N}}{10^{16} \mathrm{GeV}}\right)^{2}
$$

As figure 6 shows, if $m_{\chi}=m_{A^{\prime}}$, the lifetime of $\chi$ is smaller than one second only if $m_{A^{\prime}} \gtrsim 20 \mathrm{TeV}$. However the $\mathrm{C}$ factor above can easily be reduced by decreasing couplings and/or increasing $m_{\chi}$ with respect to $m_{A^{\prime}}$. As an example, figure 6 also gives the lifetimes keeping couplings equal to unity but taking $m_{\chi}>m_{A^{\prime}}$ and still taking the lowest value of $m_{N}$ allowed by indirect detection experiments. It shows that in this case values of $m_{A^{\prime}}$ of order the electroweak scale or below quickly become compatible both with observable neutrino and $\gamma$ fluxes and with BBN. ${ }^{9}$

\section{Comparison with other seesaw induced DM decay setups}

Concerning the decays, the main difference between the setup we consider and other scenarios where a DM decay is also induced through the seesaw interactions is that the decay width into a pair of charged lepton is suppressed by four powers of the seesaw scale rather than by two. As is well known, the Majoron, i.e. the pseudoscalar DM candidate coupling to $N \overline{N^{c}}$, not only decays into a pair of neutrinos with a width suppressed by four powers of the heavy $m_{N}$ scale, but also into a pair of charged leptons, $\ell^{+} \ell^{-}$, at the one-loop level with a width suppressed by only two powers of $m_{N}$ (from s-channel $\mathrm{Z}$ exchange and t-channel $W$-exchange diagrams, similar to those in figure 3$)[15,20,54]$. This is the result

\footnotetext{
${ }^{8}$ Another option is to assume tiny values of $Y_{L}$ and $Y_{R}$, so that the lifetime of $\chi$ is larger than the age of the Universe and also larger than indirect detection lower bounds on the lifetime. In this case the direct connection between the seesaw scale and DM lifetime is lost (and moreover this renders the lifetime of $A^{\prime}$ unobservably long).

${ }^{9}$ Actually, the $\tau_{\chi} \lesssim 1$ sec BBN constraint ought to be applied if the $\chi$ particles are numerous, as would be the case if, for instance, they decouple from the thermal bath relativistically. However, we would instead expect the $\chi$ abundance to be of the same order as the $A^{\prime}$ one, as annihilations of both particles can be dominantly driven (and Boltzmann suppressed) by the same $\mathrm{U}(1)_{X}$ gauge interactions. In this case, BBN allows for $\chi$ lifetimes a few orders of magnitude larger than $1 \mathrm{sec}$ [53], and the BBN constraint is very easily satisfied, see figure 6 .
} 


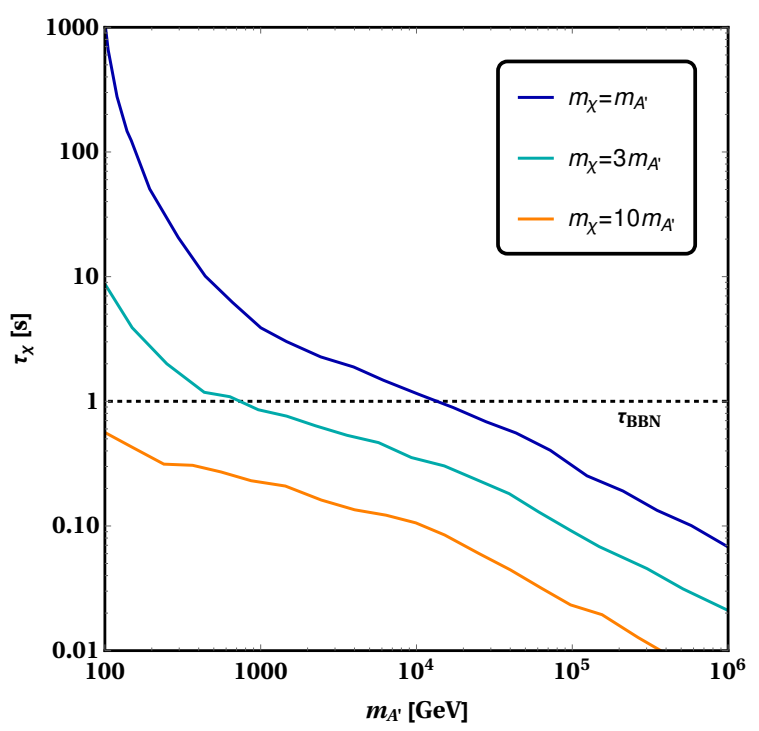

Figure 6. Lifetime of the $\chi_{1,2}$ for different ratios of $m_{\chi} / m_{A^{\prime}}$, given couplings equal to unity and the lowest value of $m_{N}$ allowed by experiments.

of the chirality flip required in the Majoron case, bringing an extra $m_{f}^{2} m_{N}^{2} / m_{W, Z}^{4}$ factor in the decay width. Although the width is greatly suppressed by the loop factor and the square of the small charged lepton mass, it still leads to much too fast a decay unless one takes the Majoron to be rather light (below $\sim 100 \mathrm{MeV}$ ) and/or we assume that the Yukawa coupling, $Y_{N}$, which leads to the masses of the right-handed neutrinos, is tiny, which implies $m_{N} \ll 10^{15} \mathrm{GeV} .{ }^{10}$ Here, instead, all decays are suppressed by four powers of the large scale $m_{N}$ and are therefore naturally enough suppressed, even for much larger DM masses. ${ }^{11}$ Thus, the production of observable energetic neutrino lines is achieved in a more straigthforward manner than for the Majoron case. For an analysis of neutrino line searches from Majoron decay, see [20].

The possibility of having a slow, seesaw-induced decay of a vector gauge boson was also studied recently in ref. [21]. The model also considers an extra $\mathrm{U}(1)^{\prime}$ gauge structure, spontaneously broken by the vev of an extra scalar, with the $\mathrm{U}(1)^{\prime}$ gauge boson being the DM particle. It also involves a neutrino portal interaction involving a singlet righthanded neutrino and an extra scalar and fermion, both charged under the $\mathrm{U}(1)^{\prime}$. As

\footnotetext{
${ }^{10}$ The interaction coupling the Majoron to a pair of right-handed neutrinos also leads to the right-handed neutrino masses, $\mathcal{L} \ni-Y_{N} \phi \overline{N^{c}} N \ni-Y_{N} f N^{c} N$ with $\phi=(f+\eta) e^{i \theta / f},\langle|\phi|\rangle=f$, and $\theta$ the Majoron. As a result, the decay width of the Majoron into a pair of charged leptons, which typically scales as $\left(m_{\nu} / v\right)^{2}\left(m_{N} / f\right)^{2}\left(m_{f} / v\right)^{2} m_{\theta}$, doesn't decrease when $m_{N}$ increases for fixed neutrino masses. Conversely, in our setup the width of $A^{\prime}$ decreases when $m_{N}$ increases, for fixed neutrino masses.

${ }^{11}$ Similarly, this is different from the decay of a $Z$ into a $b \bar{b}$ pair with a heavy top quark pair and a $W$ in the loop [55], which displays two powers of the top quark masses in the amplitude, due to the fact that for large momentum in the loop, the longitudinal $W$ exchange implies two powers of the top Yukawa couplings. In the model we consider, the $W$-exchange diagram of figure 3 in the large momentum limit instead implies two powers of $m_{A^{\prime}}$. This explains why the loop decay width is enhanced by $m_{\chi}^{4} / v^{4}$ relative to the tree-level width.
} 
mentioned above, in [21] it was emphasised that the loop contributions to the DM decay width dominate for large DM masses. This setup therefore also leads to characteristic neutrino lines from DM decays into a pair of neutrinos. The model nevertheless differs from the one we studied above in various ways. Firstly, it assumes two sets of right-handed neutrinos rather than one, a "visible" set coupling to the SM doublet of leptons (in the usual seesaw Yukawa way) and a "hidden sector" set coupling to the extra charged fermion and charged scalar. The two sets mix through tiny off-diagonal Majorana mass terms. Secondly, the extra charged fermion is chiral, rather than vector-like, and acquires its mass through a seesaw mechanism in the hidden sector. The seesaw-induced extra fermion mass is assumed not to be tiny, thereby requiring right-handed neutrinos with masses much below the Weinberg operator scale. Since the extra fermion obtains its mass via a seesaw mechanism, the one-loop induced widths of DM decays into pairs of neutrinos or charged leptons is suppressed by only two powers of the right-handed neutrino masses (rather than four as above). All this leads to too rapid a decay of the DM unless there is some tiny parameter entering into play. This is achieved by assuming that the mass mixing between the sets of right-handed neutrinos is very small. Thirdly, the chiral structure assumed requires the existence of extra fermions charged under the $\mathrm{U}(1)^{\prime}$ in order to cancel gauge anomalies.

\section{Non-abelian case}

Instead of the abelian hidden sector gauge structure above, one could have considered a non-abelian symmetry as well. The simplest possibility is a $\mathrm{SU}(2)_{X}$ gauge structure, as in [3]. In this case, this gauge symmetry is broken by a complex scalar doublet and one is left with a degenerate triplet of DM gauge bosons protected by the remnant custodial symmetry. The Lagrangian is the same as for the abelian case, eq. (2.1), provided that now $\phi$ is the doublet and the $F_{\mu \nu}$ field strength and covariant derivative stand for the $\mathrm{SU}(2)_{X}$ ones. Such a structure can also couple to the seesaw states provided that the vectorlike fermion, $\chi$, is now a doublet, in which case eq. (2.2) also holds. The DM decay phenomenology is essentially the same as for the abelian case. If $m_{\chi}>m_{\mathrm{DM}} / 2$, the nonabelian gauge bosons do not decay to a pair of $\chi$ fermions and are destabilised only by seesaw-suppressed interactions, just as in the abelian case (up to $\mathrm{SU}(2)_{X}$ combinatorial factors of order unity). In the non-abelian case there is no possibility of kinetic mixing, so that one does not need to assume that this mixing doesn't exist in order to avoid the associated fast decay.

\section{Summary}

If in a new sector a fermion singlet combination of dimension $5 / 2$ can be written down, i.e. a " $\chi \phi$ " singlet bilinear, this sector can couple to the SM through a neutrino portal interaction, $\bar{N} \chi \phi$. This induces a $\chi-\nu$ mixing mediated by a right-handed neutrino, $N$. If the DM particle in this new sector couples to the $\chi$ fermion, it can eventually decay into a final state containing ordinary neutrinos. This can lead to the emission of a striking neutrino line that can be searched for. The decay width in this case is necessarily suppressed by powers of the 
seesaw scale, i.e. by powers of the most experimentally motivated UV physical scale that we know of at the moment. This nevertheless involves the non-trivial requirement that the finite DM lifetime induced in this way is not too short, and in particular is of the order of the present experimental sensitivity. To this end, known setups of this kind typically require an additional large, ad hoc (coupling) suppression of the DM width. In this letter, we have presented examples of setups where this can be avoided, so that a DM lifetime of order the experimental sensitivity can be entirely associated with the largeness of the Weinberg operator scale, and nothing else. Given the similarity between the Weinberg operator and GUT scales, this offers the interesting possibility of probing UV physics at scales as high as the GUT scale. These results are characteristic of spin-1 DM scenarios, as considered above. Instead, a scalar or fermion DM particle gives in the simplest realisations (such as in the Majoron model or the example of the $\chi$ decay above) a lifetime suppressed by only two powers of the seesaw scale. Besides predicting neutrino lines, the spin-1 setups also predict, for large DM masses, an equal production of pairs of charged leptons.

\section{Acknowledgments}

This work is supported by the "Probing dark matter with neutrinos" ULB-ARC convention, by the F.R.S./FNRS under the Excellence of Science (EoS) project No. 30820817 - be.h "The H boson gateway to physics beyond the Standard Model", by the FRIA, and by the IISN convention 4.4503.15.

Open Access. This article is distributed under the terms of the Creative Commons Attribution License (CC-BY 4.0), which permits any use, distribution and reproduction in any medium, provided the original author(s) and source are credited.

\section{References}

[1] T. Hambye, On the stability of particle dark matter, PoS IDM2010 (2011) 098 [arXiv: 1012.4587] [INSPIRE].

[2] M. Cirelli, N. Fornengo and A. Strumia, Minimal dark matter, Nucl. Phys. B 753 (2006) 178 [hep-ph/0512090] [INSPIRE].

[3] T. Hambye, Hidden vector dark matter, JHEP 01 (2009) 028 [arXiv:0811.0172] [INSPIRE].

[4] O. Lebedev, H.M. Lee and Y. Mambrini, Vector Higgs-portal dark matter and the invisible Higgs, Phys. Lett. B 707 (2012) 570 [arXiv:1111.4482] [INSPIRE].

[5] T. Hambye and M.H.G. Tytgat, Confined hidden vector dark matter, Phys. Lett. B 683 (2010) 39 [arXiv:0907.1007] [InSPIRE].

[6] C. Arina, T. Hambye, A. Ibarra and C. Weniger, Intense Gamma-Ray Lines from Hidden Vector Dark Matter Decay, JCAP 03 (2010) 024 [arXiv:0912.4496] [INSPIRE].

[7] C. Gross, O. Lebedev and Y. Mambrini, Non-Abelian gauge fields as dark matter, JHEP 08 (2015) 158 [arXiv : 1505.07480] [inSPIRE].

[8] T. Hambye, A. Strumia and D. Teresi, Super-cool Dark Matter, JHEP 08 (2018) 188 [arXiv: 1805.01473] [INSPIRE]. 
[9] D. Buttazzo, L. Di Luzio, G. Landini, A. Strumia and D. Teresi, Dark Matter from self-dual gauge/Higgs dynamics, JHEP 10 (2019) 067 [arXiv: 1907.11228] [INSPIRE].

[10] D. Buttazzo et al., Scalar gauge dynamics and Dark Matter, JHEP 01 (2020) 130 [arXiv: 1911.04502] [INSPIRE].

[11] I.Z. Rothstein, K.S. Babu and D. Seckel, Planck scale symmetry breaking and majoron physics, Nucl. Phys. B 403 (1993) 725 [hep-ph/9301213] [INSPIRE].

[12] V. Berezinsky and J.W.F. Valle, The KeV majoron as a dark matter particle, Phys. Lett. B 318 (1993) 360 [hep-ph/9309214] [INSPIRE].

[13] M. Lattanzi and J.W.F. Valle, Decaying warm dark matter and neutrino masses, Phys. Rev. Lett. 99 (2007) 121301 [arXiv:0705.2406] [InSPIRE].

[14] F. Bazzocchi, M. Lattanzi, S. Riemer-Sørensen and J.W.F. Valle, X-ray photons from late-decaying majoron dark matter, JCAP 08 (2008) 013 [arXiv:0805.2372] [INSPIRE].

[15] M. Frigerio, T. Hambye and E. Masso, Sub-GeV dark matter as pseudo-Goldstone from the seesaw scale, Phys. Rev. X 1 (2011) 021026 [arXiv:1107.4564] [InSPIRE].

[16] M. Lattanzi, S. Riemer-Sorensen, M. Tortola and J.W.F. Valle, Updated CMB and x- and $\gamma$-ray constraints on Majoron dark matter, Phys. Rev. D 88 (2013) 063528 [arXiv: 1303.4685] [INSPIRE].

[17] F.S. Queiroz and K. Sinha, The Poker Face of the Majoron Dark Matter Model: LUX to keV Line, Phys. Lett. B 735 (2014) 69 [arXiv:1404.1400] [InSPIRE].

[18] E. Dudas, Y. Mambrini and K.A. Olive, Monochromatic neutrinos generated by dark matter and the seesaw mechanism, Phys. Rev. D 91 (2015) 075001 [arXiv:1412.3459] [INSPIRE].

[19] W. Wang and Z.-L. Han, Global U(1) $)_{L}$ Breaking in Neutrinophilic 2HDM: From LHC Signatures to X-Ray Line, Phys. Rev. D 94 (2016) 053015 [arXiv: 1605.00239] [InSPIRE].

[20] C. Garcia-Cely and J. Heeck, Neutrino Lines from Majoron Dark Matter, JHEP 05 (2017) 102 [arXiv: 1701.07209] [INSPIRE].

[21] H.H. Patel, S. Profumo and B. Shakya, Loop dominated signals from neutrino portal dark matter, Phys. Rev. D 101 (2020) 095001 [arXiv:1912.05581] [INSPIRE].

[22] M. Srednicki, S. Theisen and J. Silk, Cosmic Quarkonium: A Probe of Dark Matter, Phys. Rev. Lett. 56 (1986) 263 [Erratum ibid. 56 (1986) 1883] [INSPIRE].

[23] L. Bergstrom and H. Snellman, Observable Monochromatic Photons From Cosmic Photino Annihilation, Phys. Rev. D 37 (1988) 3737 [InSPIRE].

[24] S. Rudaz, On the Annihilation of Heavy Neutral Fermion Pairs Into Monochromatic gamma-rays and Its Astrophysical Implications, Phys. Rev. D 39 (1989) 3549 [INSPIRE].

[25] A. Bouquet, P. Salati and J. Silk, $\gamma$-Ray Lines as a Probe for a Cold Dark Matter Halo, Phys. Rev. D 40 (1989) 3168 [inSPIRE].

[26] S. Rudaz and F.W. Stecker, On the Observability of the $\gamma$ Ray Line Flux From Dark Matter Annihilation, Astrophys. J. 368 (1991) 406 [INSPIRE].

[27] L. Bergstrom, P. Ullio and J.H. Buckley, Observability of gamma-rays from dark matter neutralino annihilations in the Milky Way halo, Astropart. Phys. 9 (1998) 137 [astro-ph/9712318] [INSPIRE]. 
[28] M. Gustafsson, T. Hambye and T. Scarna, Effective Theory of Dark Matter Decay into Monochromatic Photons and its Implications: Constraints from Associated Cosmic-Ray Emission, Phys. Lett. B $\mathbf{7 2 4}$ (2013) 288 [arXiv: 1303.4423] [INSPIRE].

[29] P. Baratella, M. Cirelli, A. Hektor, J. Pata, M. P IIBeleht and A. Strumia, PPPC \& DMv: $a$ Poor Particle Physicist Cookbook for Neutrinos from Dark Matter annihilations in the Sun, JCAP 03 (2014) 053 [arXiv: 1312.6408] [INSPIRE].

[30] C. El Aisati, M. Gustafsson and T. Hambye, New Search for Monochromatic Neutrinos from Dark Matter Decay, Phys. Rev. D 92 (2015) 123515 [arXiv:1506.02657] [inSPIRE].

[31] C. El Aisati, M. Gustafsson, T. Hambye and T. Scarna, Dark Matter Decay to a Photon and a Neutrino: the Double Monochromatic Smoking Gun Scenario, Phys. Rev. D 93 (2016) 043535 [arXiv: 1510.05008] [inSPIRE].

[32] C. El Aisati, C. Garcia-Cely, T. Hambye and L. Vanderheyden, Prospects for discovering a neutrino line induced by dark matter annihilation, JCAP 10 (2017) 021 [arXiv:1706.06600] [INSPIRE].

[33] Y. Chikashige, R.N. Mohapatra and R.D. Peccei, Are There Real Goldstone Bosons Associated with Broken Lepton Number?, Phys. Lett. B 98 (1981) 265 [INSPIRE].

[34] J. Schechter and J.W.F. Valle, Neutrino Decay and Spontaneous Violation of Lepton Number, Phys. Rev. D 25 (1982) 774 [INSPIRE].

[35] N. Bernal, X. Chu, C. Garcia-Cely, T. Hambye and B. Zaldivar, Production Regimes for Self-Interacting Dark Matter, JCAP 03 (2016) 018 [arXiv:1510.08063] [INSPIRE].

[36] S. Palomares-Ruiz and S. Pascoli, Testing MeV dark matter with neutrino detectors, Phys. Rev. D 77 (2008) 025025 [arXiv:0710.5420] [INSPIRE].

[37] S. Palomares-Ruiz, Model-independent bound on the dark matter lifetime, Phys. Lett. B 665 (2008) 50 [arXiv: 0712.1937] [INSPIRE].

[38] Super-Kamiokande collaboration, Indirect searches for dark matter particles with the Super-Kamiokande detector, Nuovo Cim. C 38 (2016) 125 [INSPIRE].

[39] ICECuBE collaboration, Search for neutrinos from decaying dark matter with IceCube, Eur. Phys. J. C 78 (2018) 831 [arXiv: 1804.03848] [INSPIRE].

[40] IceCube collaboration, Indirect Dark Matter Searches with IceCube, J. Phys. Conf. Ser. 1342 (2020) 012074 [inSPIRE].

[41] Borexino collaboration, Study of solar and other unknown anti-neutrino fluxes with Borexino at LNGS, Phys. Lett. B 696 (2011) 191 [arXiv:1010.0029] [INSPIRE].

[42] KamLAND collaboration, A study of extraterrestrial antineutrino sources with the KamLAND detector, Astrophys. J. $\mathbf{7 4 5}$ (2012) 193 [arXiv:1105.3516] [INSPIRE].

[43] Super-Kamiokande collaboration, Search for supernova relic neutrinos at Super-Kamiokande, Phys. Rev. Lett. 90 (2003) 061101 [hep-ex/0209028] [INSPIRE].

[44] Super-Kamiokande collaboration, Supernova Relic Neutrino Search with Neutron Tagging at Super-Kamiokande-IV, Astropart. Phys. 60 (2015) 41 [arXiv:1311.3738] [INSPIRE].

[45] T. Cohen, K. Murase, N.L. Rodd, B.R. Safdi and Y. Soreq, $\gamma$-ray Constraints on Decaying Dark Matter and Implications for IceCube, Phys. Rev. Lett. 119 (2017) 021102 [arXiv: 1612.05638] [INSPIRE]. 
[46] V. Poulin, P.D. Serpico and J. Lesgourgues, A fresh look at linear cosmological constraints on a decaying dark matter component, JCAP 08 (2016) 036 [arXiv: 1606.02073] [INSPIRE].

[47] K. Murase and J.F. Beacom, Constraining Very Heavy Dark Matter Using Diffuse Backgrounds of Neutrinos and Cascaded Gamma Rays, JCAP 10 (2012) 043 [arXiv:1206.2595] [INSPIRE].

[48] C. Blanco and D. Hooper, Constraints on Decaying Dark Matter from the Isotropic Gamma-Ray Background, JCAP 03 (2019) 019 [arXiv: 1811.05988] [INSPIRE].

[49] ICECuBE collaboration, Constraints on the Extremely-high Energy Cosmic Neutrino Flux with the IceCube 2008-2009 Data, Phys. Rev. D 83 (2011) 092003 [Erratum ibid. 84 (2011) 079902] [arXiv:1103.4250] [INSPIRE].

[50] Fermi-LAT collaboration, The Spectrum of the Isotropic Diffuse Gamma-Ray Emission Derived From First-Year Fermi Large Area Telescope Data, Phys. Rev. Lett. 104 (2010) 101101 [arXiv: 1002.3603] [INSPIRE].

[51] Fermi-LAT collaboration, Constraints on the Galactic Halo Dark Matter from Fermi-LAT Diffuse Measurements, Astrophys. J. 761 (2012) 91 [arXiv:1205.6474] [INSPIRE].

[52] FERMI-LAT collaboration, The spectrum of isotropic diffuse gamma-ray emission between $100 \mathrm{MeV}$ and $820 \mathrm{GeV}$, Astrophys. J. 799 (2015) 86 [arXiv:1410.3696] [INSPIRE].

[53] M. Hufnagel, K. Schmidt-Hoberg and S. Wild, BBN constraints on MeV-scale dark sectors. Part II. Electromagnetic decays, JCAP 11 (2018) 032 [arXiv:1808.09324] [INSPIRE].

[54] A. Pilaftsis, Astrophysical and terrestrial constraints on singlet Majoron models, Phys. Rev. D 49 (1994) 2398 [hep-ph/9308258] [INSPIRE].

[55] J. Bernabeu, A. Pich and A. Santamaria, $\Gamma(Z \rightarrow B \bar{B}):$ A Signature of Hard Mass Terms for a Heavy Top, Phys. Lett. B 200 (1988) 569 [inSPIRE]. 